\title{
Docking-based strategy to design novel flavone-based arylamides as potent V600E-BRAF inhibitors with prediction of their drug-likeness and ADMET properties
}

\author{
Abdullahi Bello Umar , Adamu Uzairu, Gideon Adamu Shallangwa and Sani Uba
}

\begin{abstract}
Background: V600E-BRAF protein target has much potential for scientific research as therapeutic target due to its involvement in human melanoma cancer. In the current research, molecular docking investigation was conducted on some flavone-based arylamides as anticancer drug candidates via V600E-BRAF inhibition with the help of docking software Molegro Virtual Docker. Based on the predicted results, existing structures were modified and screened for pharmacokinetics ADMET properties.

Results: The docking result demonstrates that compound 28 best inhibits V600E-BRAF when compared with other compounds within the dataset. This compound was used as a template in designing novel anticancer compounds by attaching some favorable substituents. The docking results of the designed compounds revealed a good MolDock score $(<-90)$, which showed that all the compounds can efficiently bind with the active sites of the target, out of which two analogous (N1 and N3) were considered optimal that outperformed vemurafenib, the FDA-approved V600E-BRAF inhibitor. Furthermore, these compounds passed the drug-likeness criteria (Lipinski's rule) successfully and were found to be orally bioavailable. Also, the designed compounds were found to have good pharmacokinetics absorption, distribution, metabolism, excretion, and toxicity (ADMET) properties.

Conclusion: Thus, this study identified compounds (N1 and N3) as the best hits against V600E-BRAF kinase with enhanced pharmacological properties and recommends their synthesis, in vivo and ex vivo evaluation to validate our hypothesis.
\end{abstract}

Keywords: Melanoma, V600E-BRAF, Virtual screening, Docking simulation, ADMET

\section{Background}

The BRAF kinase is a proto-oncogene that activates the MAP kinase pathway, and its mutations are extremely widespread and are mostly seen in nearly $66 \%$ of melanomas (Kudchadkar et al. 2012; Salton et al. 2015). The most popular melanoma mutation is V600E-BRAF that constitutively stimulates downstream MAPK signaling (Wan et al. 2004). Melanoma is one of the rapidly growing

\footnotetext{
*Correspondence: abdallahbum@gmail.com

Physical Chemistry Research Group, Faculty of Physical Sciences, Ahmad Bello University, Zaria, Nigeria
}

forms of skin tumor, problematic and its advanced (metastatic) stage carries high mortality rates as a result of their development of resistance to the known drugs used to treat melanoma (Gray-Schopfer et al. 2007). For example, vemurafenib (PLX4032) was clinically approved in 2011 by the FDA for metastatic melanoma and primarily directed towards the V600E-BRAF mutation (Robinson et al. 2014). This has substantially increased the efficacy with a total response rate of about $48 \%$ when compared with chemotherapy which is about $5 \%$ using dacarbazine (Chapman et al. 2011). However, most patients administered with vemurafenib end up developing resistance 
toward it. Therefore, identifying other V600E-BRAF kinase inhibitors is very significant for cancer research (Roskoski 2012).

Several methods have been used to improve the existing anticancer drugs such as the formulation of nanomedicines (Elnaggar et al. 2019). Nevertheless, knowing the tumors' heterogeneity and the development of resistance provide the evolvement of novel anticancer agents as an urgent medical necessity. The arylamide derivatives are an attractive class of organic compounds possessing various biological activities (Landucci et al. 2019; $\mathrm{Vu}$ et al. 2016). Various arylamide derivatives were developed and used as potential anticancer agents acting on several molecular targets and even sometimes with yet unknown molecular targets. Flavones consist of an attractive category of natural products possessing several biological activities (Zhao et al. 2019; Verma and Pratap 2010). Their antiproliferative activities have been reported to involve multiple mechanisms of action including autophagy induction, apoptosis, cell cycle suppression, and inhibition of many signaling pathways like PI3K/Akt and MAPK, as well as inhibition of calcium channels (Zhang et al. 2017; Borah et al. 2017).

In the drug discovery process, promising lead identification is accomplished by high-throughput experimental screening (HTS), but it is time-consuming and costly (Cheng et al. 2012). Therefore, it is necessary to overcome these limitations of conventional drug identification approaches with low cost, effective, and broad-spectrum computational approaches. High-throughput in silico virtual screening, which is extensively employed presently in many pharmaceutical industry research units, is a screening approach generally utilized by the medicinal chemist. The accessibility of the crystal structure of the protein receptor as a template for the in silico screening increases the chances of virtual screening (Luo et al. 2008; Schmidt et al. 2014). In this study, thirty-one (31) flavone-based aryl amides were retrieved from the work of Hassan et al. (2020). The compounds were then docked in the ATPactive site of the V600E-BRAF kinase (PDB ID: 3O7G), a known melanoma target (Dhillon et al. 2007) utilizing Molegro Virtual Docker (MVD) (Molegro 2011) (version 6.0). After conducting virtual screening alongside scaffold growth, new compounds were developed and ranked based on their docking score and binding modes. Additionally, the new compounds were screened via Lipinski's rule of five for oral bioavailability and pharmacokinetics ADMET properties.

\section{Methods}

\section{Collection of ligands and optimization}

A series of thirty-one (31) flavone-based arylamides presented in Additional file 1: Table S1, as potential
V600E-BRAF kinase inhibitors were selected from the literature (Hassan et al. 2020). The two-dimensional chemical structural forms of the collected compounds have been drawn with ChemDraw V12.0 and transformed into three-dimensional chemical structural forms and optimize their geometry by adopting density functional theory (DFT) approach at the B3LYP level of theory and 6-31G*: as the basis set with Spartan 14 v. 1.1.4 software package from Wavefunction Inc. The fully optimized structures of the inhibitors were stored in PDB format for docking utilizing Spartan 14 and then taken to the Molegro-Virtual-Docker (MVD) (Molegro 2011), for the docking purposes.

\section{Protein preparation and molecular docking simulation}

The 3D X-ray structure of the receptor (V600E-BRAF) attached with vemurafenib (PDB-ID: 3OG7) (Brose et al. 2002; Bollag et al. 2010; Choi et al. 2011) was collected from the Protein Databank at (https://www.rcsb. org/). The PDB file of V600E-BRAF was prepared using Molegro Virtual Docker 6.0 (Molegro 2011) by updating the $\mathrm{H}$-atoms and removal of the water (excess) molecules found in the complex structure, and the attached ligand (vemurafenib) was also removed from the target. The potential ligand-binding cavity of the V600E-BRAF receptor was predicted, and the binding cavity was set inside a restricted sphere of $\mathrm{X}: 1.59, \mathrm{Y}:-1.28, \mathrm{Z}:-6.21$ with a radius $28 \AA$ having a grid resolution of $0.30 \AA$.

For molecular docking, all the prepared compounds (ligands) including vemurafenib (reference inhibitor) were then imported into the Molegro Virtual Docker 6.0 and its bond flexibility was set together with the side chains of the amino acid, which was also set inside the restricted sphere. The flexibility was set with a strength of 0.90 and a tolerance of 1.10 . The RMSD threshold was set as $2.00 \AA$ for the multiple clusters poses with 100.00 energy penalty values. The docking algorithm was set for a maximum of 1500 iteration with a simplex evolution size of 50. The docking simulation was run for a minimum of 50 times for the 10 poses, and the best poses were chosen based on the set scoring functions such as the MolDock score, Rerank score, E-interaction, and E-H bond (Thomsen and Christensen 2006). Additionally, Discovery Studio (DS) Visualizer v. 3.5 was adopted to visualize various intermolecular interactions such as $\mathrm{H}$ bond, hydrophobic, halo-bond, and $\pi /$ aryl interactions.

\section{Prediction of pharmacokinetics (ADMET) and drug-likeness properties}

The application of computational tools for identifying the novel drug candidate assists to lessen the number of experimental researches and for increasing the success rate. For this purpose, we applied Lipinski's rule of five 
for drug-likeness as an initial screening step for oral bioavailability and synthetic accessibility using SwissADME (www.swissadme.ch/) online tool.

Next, secondary screening was conducted by calculating the ADMET properties as a measure of the pharmacokinetics (Martinez-Mayorga et al. 2020) using pkCSM (https://biosig.unimelb.edu.au/pkcsm//) online tool.

\section{Results}

The docking scores were served in the form MolDock and Rerank score respectively as presented in Additional file 1: Table S2. The optimal V600E-BRAF inhibitors were sorted based on the highest docking score, and most of the screened compounds are efficient in targeting V600EBRAF. Also, to give a better understanding of the excellent docking score observed, we examined the interaction of the most potent inhibitor (compound 28) with the V600E-BRAF. The docked pose of compound 28 is shown in Fig. 1 and is selected as a template to design new molecules as in Fig. 2 showing $\mathbf{R}_{\mathbf{1}}$ and $\mathbf{R}_{\mathbf{2}}$ positions as a point of attachment. The detailed docking results and types of interactions involved in the selected template (compound 28), designed compounds, and vemurafenib as reference inhibitor are presented in Tables 1 and 2, respectively.

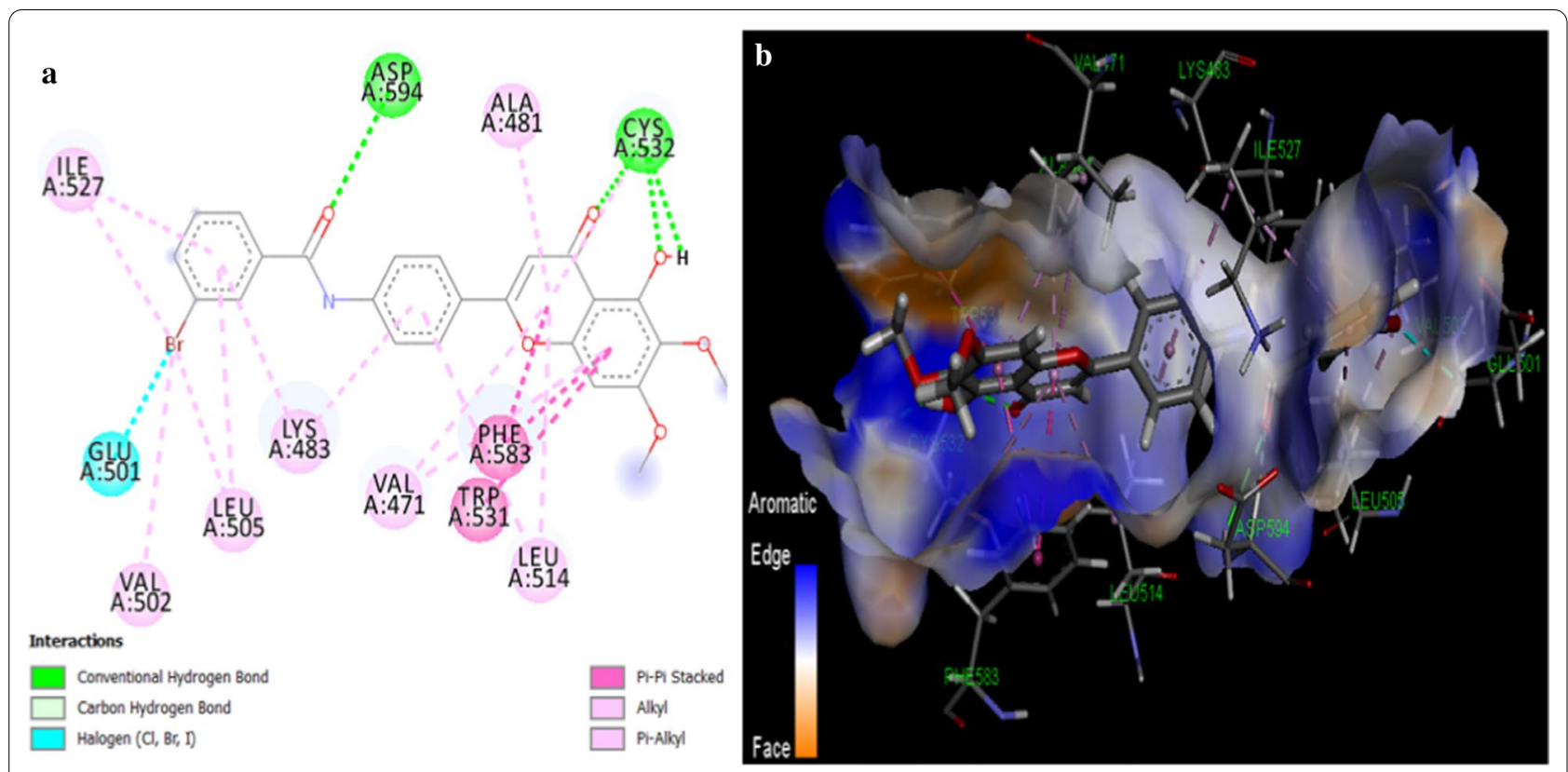

Fig. 1 a 2D diagram for the interaction of compound 28 with V600E-BRAF, b 3D view of the compound 28 in the binding site of V600E-BRAF

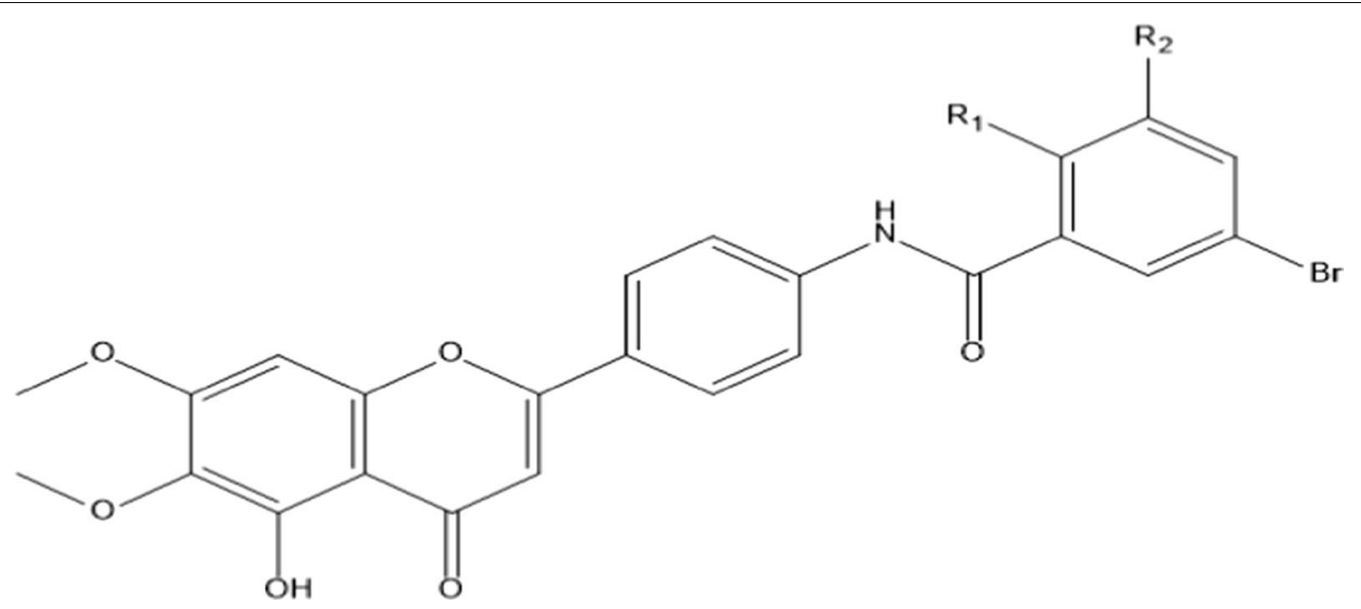

Fig. 2 Structural skeleton of compound 28 showing $R_{1}$ and $R_{2}$ positions as a point of attachment 
Table 1 The Docking results of compound 28, designed compounds with vemurafenib as reference

\begin{tabular}{lllll}
\hline SN & MolDock score $^{\mathbf{a}}$ & Rerank score $^{\mathbf{b}}$ & E-inter $^{\mathbf{c}}$ & E-H bond $^{\mathbf{d}}$ \\
\hline 28 & -160.581 & -135.878 & -185.831 & -4.387 \\
N1 & -164.145 & -126.882 & -173.404 & -5.098 \\
N2 & -148.244 & -98.702 & -157.235 & -4.021 \\
N3 & -166.413 & -141.170 & -185.847 & -4.834 \\
Vem & -158.139 & -118.607 & -167.952 & -4.741 \\
\hline
\end{tabular}

${ }^{a}$ Moldock score was obtained from the PLP scoring functions with a new $\mathrm{H}$-bond term and extra charge schemes (Molegro 2011)

${ }^{b}$ Rerank score is a linear combination of E-inter (electrostatic, van der Waals, $\mathrm{H}$-bonding, steric) between the ligand and the protein target, and $\mathrm{E}$-intra (electrostatic, van der Waals, H-bonding, sp2-sp2, torsion) of the ligand weighted by pre-defined coefficients (Molegro 2011)

c $\mathrm{E}$-inter is the total interaction energy between the protein and the pose

${ }^{d} \mathrm{E}-\mathrm{H}$ bond is $\mathrm{H}$ bond energy

Figures 3, 4, 5, and 6 depict the 2D view and 3D view of the docked poses of compound 28, designed compounds $(\mathrm{N} 1, \mathrm{~N} 2$, and N3) and vemurafenib, respectively. Moreover, to ensure that the designed compounds are the viable drugs, the drug-likeness, and pharmacokinetics ADMET properties were evaluated with vemurafenib as the reference. The SwissADME online tool was used to predict the drug-likeness properties as presented in Table 3, and the pkCSM online tool was adopted in predicting the ADMET properties reported in Table 4.

\section{Discussion}

In the current research, docking simulation was applied repetitiously to identify the optimal V600E-BRAF inhibitors, in which docking scores were served in the form MolDock and Rerank score, respectively. The optimal V600E-BRAF inhibitors were sorted based on the highest docking score, and most of the screened compounds are efficient in targeting V600E-BRAF. From the docking result reported in Additional file 1: Table S2, it was found that all the studied compounds formed bonds and non-bond interaction at the binding pocket of the receptor as manifest from the E-interaction and E-H bond. The compounds pose maximum MolDock Score as well as the Rerank score, indicating that they had favorable ligand-protein interaction comparable to vemurafenib at the binding site of V600E-BRAF. Also, to give a better understanding of the excellent docking score observed, we examined the interaction of the most potent inhibitor (compound 28) with the V600E-BRAF.

Compound 28 inhibits V600E-BRAF with a MolDock and Rerank score of -160.581 and -135.878 , respectively, while the E-H bond was -4.387 (Table 1). As shown in Fig. 1, the good binding score observed was due to the formation of four $\mathrm{H}$-bond, one $\mathrm{C}-\mathrm{H}$ bond interaction, and three $\pi-\pi$ stacked interaction. The carbonyl $\mathrm{O}$-atom of the chromen ring, $\mathrm{O}$-atom, and $\mathrm{H}$-atom of hydroxyl group attached to the benzene ring formed three $\mathrm{H}$ bond with CYS532. Another $\mathrm{H}$ bond is formed from the carbonyl $\mathrm{O}$-atom of the acetamide linker to ASP594 residue. There is a formation of one $\mathrm{C}-\mathrm{H}$ bond with ASP594 residue. Moreover, the two rings of chromene moiety intercalated in space to form five $\pi-\pi$ interaction (stacked) with TRP531 and PHE583 residues similar to vemurafenib as presented (Table 2). There is a formation of one halogen bond between the $\mathrm{Br}$ atom of the benzene ring and GLU501 residue. The surface mode of the receptor shown in Fig. $1 \mathrm{~b}$ indicates that compound 28 has a good orientation at the ATP binding site of V600E-BRAF (Umar et al. 2020). Besides, the good docking score of compound 28 observed may also be connected to some other weak interactions, such as $\pi$-alkyl interaction as presented in Fig. 1.

As observed from the docked pose of compound 28 (Fig. 1), there is still enough margin of a gap to be used. From the view of strong potency, the interior surface slot ought to be occupied to give better binding potency and more interactions, and this can be achieved if the selected compound could be optimized by some favorable substituents. Structural skeleton of compound 28 as a template to develop scaffolds to obtain new compounds as V600EBRAF inhibitors is presented in Fig. 2. Then, analyzing the results of docking and molecular properties, some favorable substituents were added to the selected template at $\mathbf{R}_{\mathbf{1}}$ and $\mathbf{R}_{\mathbf{2}}$ positions. In the present research, this was performed automatically using the Scaffold Grow module in the Discovery Studio and followed by docking screening using MVD. After conducting molecular docking simulation for the designed compounds, it was observed that the docking score of the selected template (compound 28) has increased as presented in Table 1. Therefore, these new compounds are novel inhibitors of V600E-BRAF, and their docking results are compared to the docking results of compound 28 and vemurafenib as a positive control. Also, to give a better interpretation of the excellent docking scores observed, the interaction of the designed compounds was examined. The detailed docking results and types of interactions involved are presented in Tables 1 and 2, respectively. Additionally, all the designed compounds have a MolDock score $<-90$, which indicates that the novel inhibitors can bind the target efficiently (Abdullahi et al. 2020).

The binding mode of $\mathrm{N} 1$ on the V600E-BRAF receptor is depicted in Fig. 3, which has a MolDock and Rerank score of -164.145 and -126.882 , respectively, while the $\mathrm{E}-\mathrm{H}$ bond was -5.098 (Table 1). It is bonded into the V600E-BRAF binding cavity via four $\mathrm{H}$ bond and three $\pi-\pi$ stacked interaction. The carbonyl O-atom 
Table 2 Molecular interactions present in the docked poses and the amino acids involved

\begin{tabular}{|c|c|c|c|c|c|c|c|c|c|}
\hline SN & $\mathrm{H}$ bond & $\begin{array}{l}\text { Bond length }(\AA) \\
\text { for } \mathrm{H} \text { bond }\end{array}$ & $\mathrm{C}-\mathrm{H}$ bond & Alkyl & Pi-alkyl & $\mathrm{Pi}-\mathrm{Pi}$ & Pi-cation & Pi-sulfur/sigma & Halo-bond \\
\hline 28 & $\begin{array}{l}\text { CYS532 } \\
\text { CYS532 } \\
\text { ASP594 } \\
\text { CYS532 }\end{array}$ & $\begin{array}{l}2.43662 \\
2.28681 \\
2.80928 \\
2.22688\end{array}$ & ASP594 & $\begin{array}{l}\text { VAL502 } \\
\text { LEU505 } \\
\text { ILE527 }\end{array}$ & $\begin{array}{l}\text { LYS483 } \\
\text { LEU505 } \\
\text { ILE527 } \\
\text { LYS483 } \\
\text { LEU514 } \\
\text { VAL471 } \\
\text { ALA481 } \\
\text { LEU514 } \\
\text { CYS532 } \\
\text { VAL471 }\end{array}$ & $\begin{array}{l}\text { TRP531 } \\
\text { PHE583 } \\
\text { PHE583 }\end{array}$ & & & GLU501 \\
\hline N1 & $\begin{array}{l}\text { CYS532 } \\
\text { CYS532 } \\
\text { GLY596 } \\
\text { CYS532 }\end{array}$ & $\begin{array}{l}2.32682 \\
2.28851 \\
2.81173 \\
2.25834\end{array}$ & & $\begin{array}{l}\text { LYS483 } \\
\text { VAL502 } \\
\text { LEU505 } \\
\text { ILE527 }\end{array}$ & $\begin{array}{l}\text { PHE468 } \\
\text { PHE498 } \\
\text { LEU505 } \\
\text { LYS483 } \\
\text { LEU514 } \\
\text { VAL471 } \\
\text { ALA481 } \\
\text { LEU514 } \\
\text { CYS532 } \\
\text { VAL471 } \\
\text { ALA481 }\end{array}$ & $\begin{array}{l}\text { TRP531 } \\
\text { PHE583 } \\
\text { PHE583 }\end{array}$ & & ILE527 & $\begin{array}{l}\text { GLU501 } \\
\text { ASP594 }\end{array}$ \\
\hline N2 & $\begin{array}{l}\text { SER536 } \\
\text { ASP594 } \\
\text { ILE527 }\end{array}$ & $\begin{array}{l}2.81389 \\
1.5465 \\
2.40925\end{array}$ & $\begin{array}{l}\text { SER535 } \\
\text { GLY593 } \\
\text { GLY534 }\end{array}$ & & $\begin{array}{l}\text { VAL471 } \\
\text { ALA481 } \\
\text { CYS532 } \\
\text { VAL471 } \\
\text { ALA481 } \\
\text { LYS483 } \\
\text { LEU514 } \\
\text { LYS483 } \\
\text { LEU514 }\end{array}$ & TRP531 & LYS483 & GLY534 & \\
\hline N3 & $\begin{array}{l}\text { ASP594 } \\
\text { PHE595 } \\
\text { GLY596 } \\
\text { GLY534 }\end{array}$ & $\begin{array}{l}2.49798 \\
2.78101 \\
1.90411 \\
2.04787\end{array}$ & $\begin{array}{l}\text { SER535 } \\
\text { GLY593 } \\
\text { ASP594 } \\
\text { GLY596 } \\
\text { HIS539 }\end{array}$ & $\begin{array}{l}\text { LEU505 } \\
\text { ILE527 } \\
\text { LYS483 } \\
\text { ILE527 }\end{array}$ & $\begin{array}{l}\text { LYS483 } \\
\text { LEU514 } \\
\text { ALA481 } \\
\text { CYS532 }\end{array}$ & PHE583 & LYS483 & & \\
\hline Vem & $\begin{array}{l}\text { CYS532 } \\
\text { GLY596 } \\
\text { GLN530 }\end{array}$ & $\begin{array}{l}1.69013 \\
2.06126 \\
1.74262\end{array}$ & $\begin{array}{l}\text { ASP594 } \\
\text { GLY596 } \\
\text { CYS532 } \\
\text { THR529 }\end{array}$ & $\begin{array}{l}\text { LEU505 } \\
\text { ILE527 }\end{array}$ & $\begin{array}{l}\text { PHE595 } \\
\text { LYS483 } \\
\text { ALA481 } \\
\text { LEU514 } \\
\text { CYS532 } \\
\text { ALA481 } \\
\text { CYS532 }\end{array}$ & $\begin{array}{l}\text { TRP531 } \\
\text { PHE583 }\end{array}$ & LYS483 & & ALA481 \\
\hline
\end{tabular}

$\mathrm{H}$ bond hydrogen bond, $\mathrm{C}-\mathrm{H}$ bond carbon hydrogen bond, Halo-bond halogen bond

of the chromen ring, $\mathrm{O}$-atom, and $\mathrm{H}$-atom of hydroxyl group attached to the benzene ring formed three $\mathrm{H}$ bond with CYS532. Another $\mathrm{H}$ bond is formed from the carbonyl O-atom of the acetamide linker to ASP594 residue. Similarly, another one was formed between the $\mathrm{N}$-atom of the $-\mathrm{NH}_{2}$ group linked to the benzene ring to GLY596 residue. Moreover, the two rings of the chromen moiety have intercalated to form a $\pi-\pi$ interaction with TRP531 and PHE583 residues. There is a formation of one $\pi$-sigma interaction between the benzene ring and ILE527 residue. Two halogen bond interaction was observed between the $\mathrm{Br}$ and $\mathrm{Cl}$ atoms attached to the benzene ring and GLU501 and ASP594 residues, respectively. Additionally, the good docking score of N1 observed may be linked to other weak interactions: alkyl with LYS483, VAL502, LEU505, and ILE527, and $\pi$-alkyl interaction with PHE468, PHE498, LEU505, LYS483, LEU514, VAL471, ALA481, LEU514, CYS532, VAL471, and ALA481 residues, respectively. The 3D protein surface shown in Fig. 2b implied that $\mathrm{N} 1$ has a good orientation in the ATP-binding cavity of V600E-BRAF kinase.

N2 docked in the binding site of V600E-BRAF (Fig. 4) showed a good docking score (Table 1), indicating that it has bound into the binding site of one of the protomers in the protein dimer via the formation of three $\mathrm{H}$ bond with SER536, ASP594, and ILE527. The O-atom of the nitro 


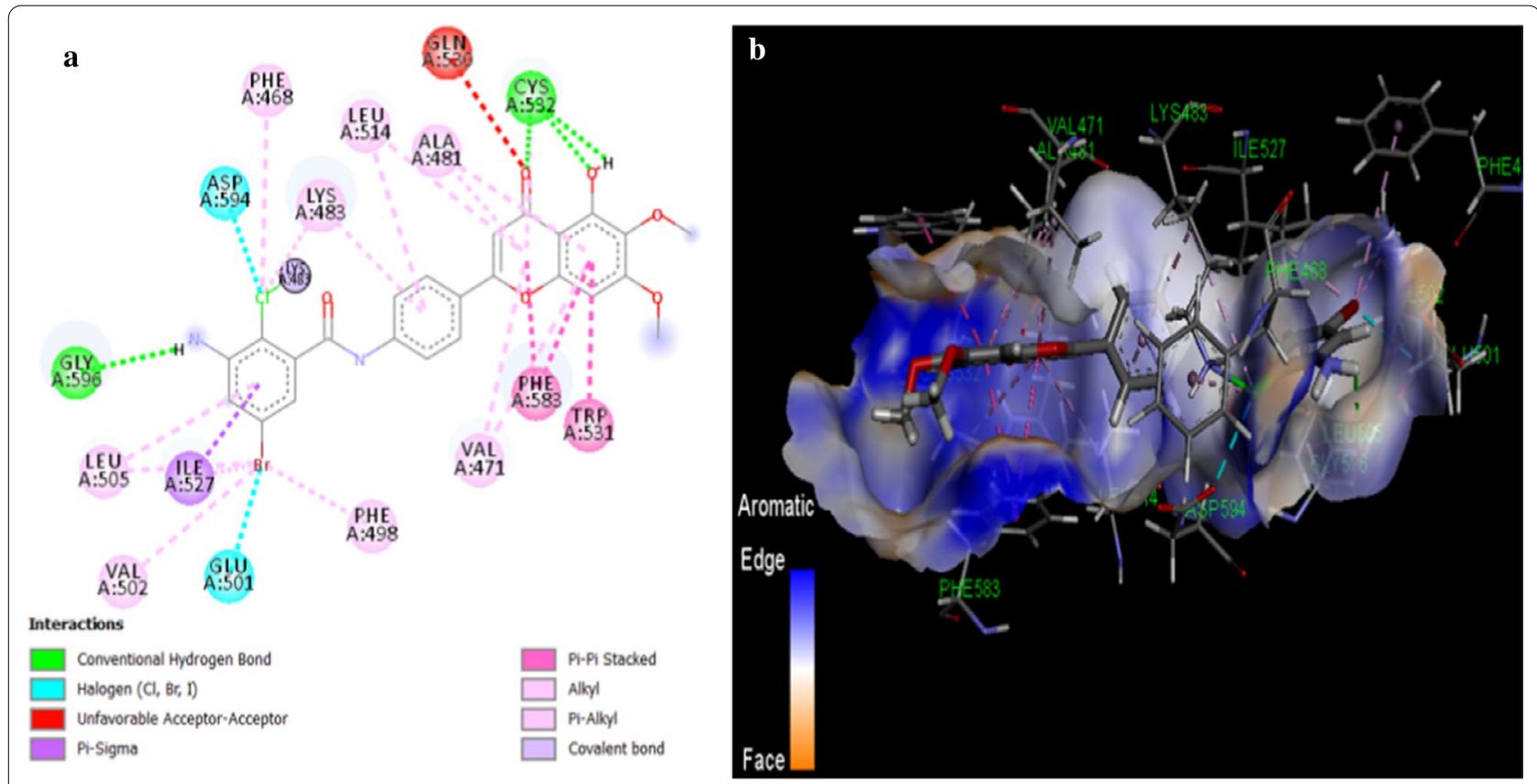

Fig. 3 a 2D diagram for the interaction of compound N1 with V600E-BRAF, b 3D view of the compound N1 in the binding site of V600E-BRAF

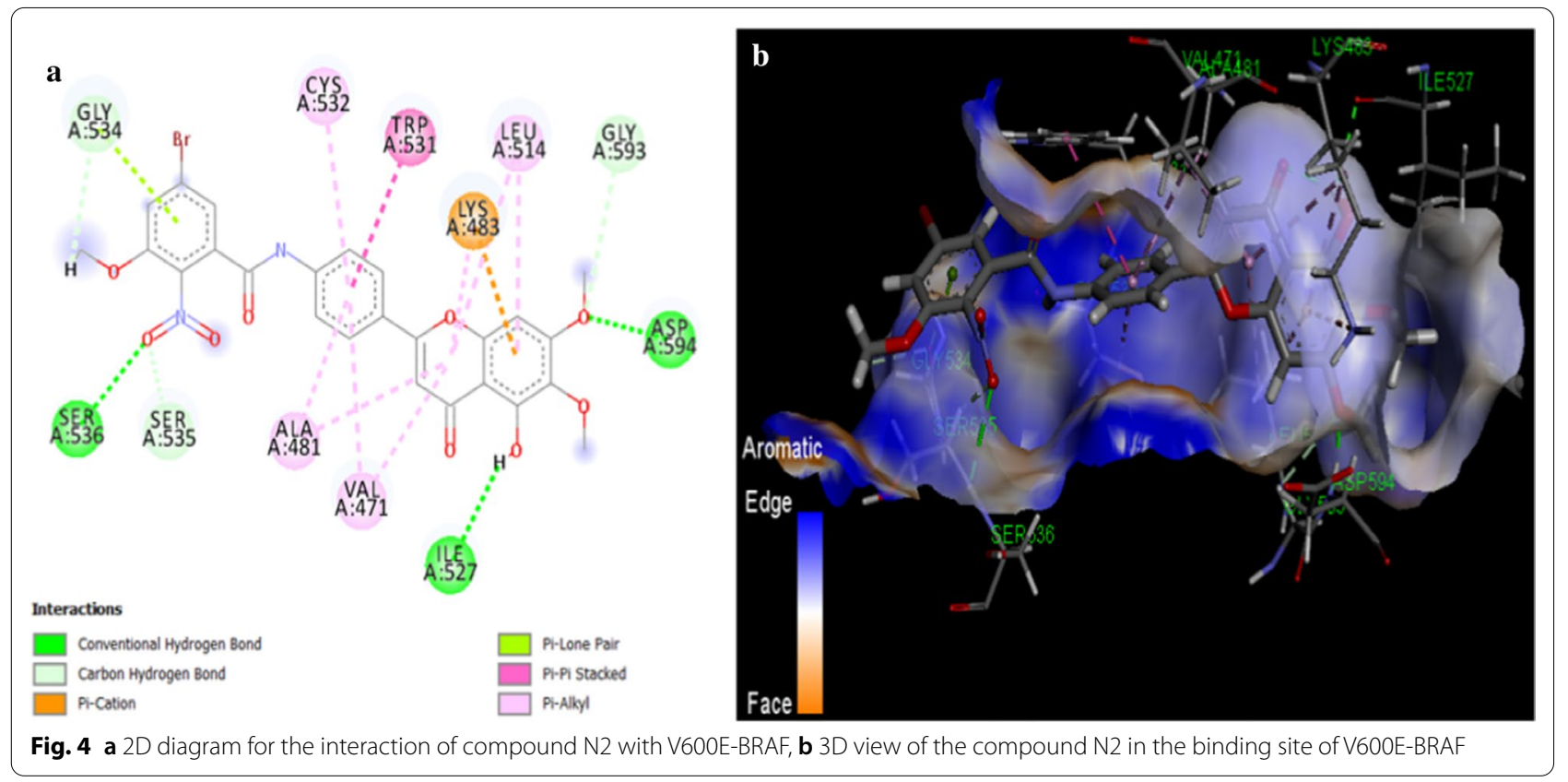

group at $\mathrm{p}$ - of benzene ring moiety formed one $\mathrm{H}$ bond with SER536, and the second bond was formed between the $-\mathrm{OCH}_{3}$ group attached with the chromene ring and ASP594. The other one was formed between the $\mathrm{OH}$ group to ILE527 residue. Four $\mathrm{C}-\mathrm{H}$ bond were observed with SER535 GLY593 GLY534 residues in the complex. Further, there was $\pi-\pi$ interaction (staked) from the active site and the compound to with TRP531 residue, which occurs due to the intercalation of the 'benzene ring (Fig. 3). There is a formation of one $\pi$-cation interaction between from the chromen ring moiety to LYS483 residue and lastly $\pi$-alkyl interaction with four residues (VAL471, ALA481, CYS532, VAL471, ALA481, LYS483, LEU514, LYS483, and LEU514). The docking results in 


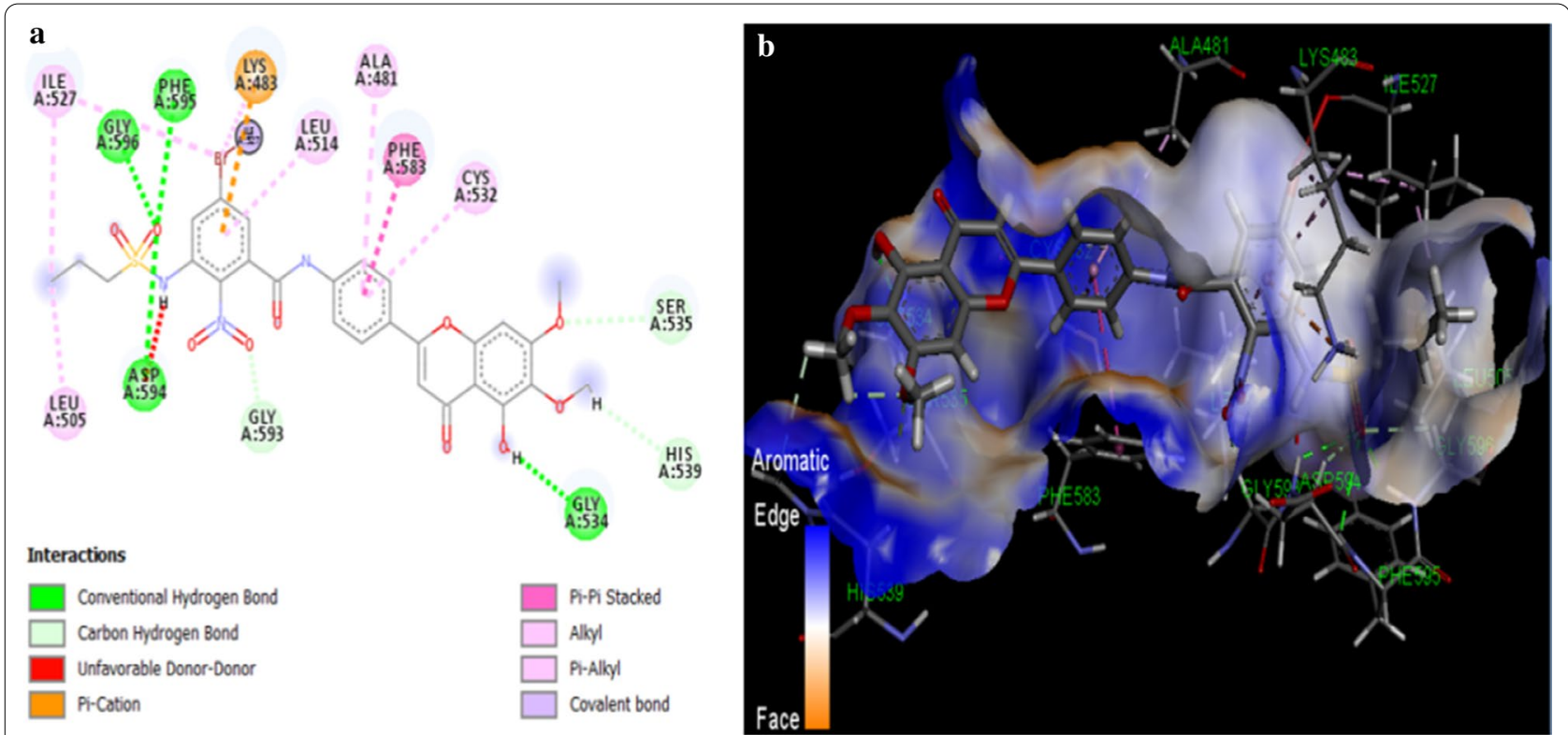

Fig. 5 a 2D diagram for the interaction of compound N3 with V600E-BRAF, b 3D view of the compound N3 in the binding site of V600E-BRAF

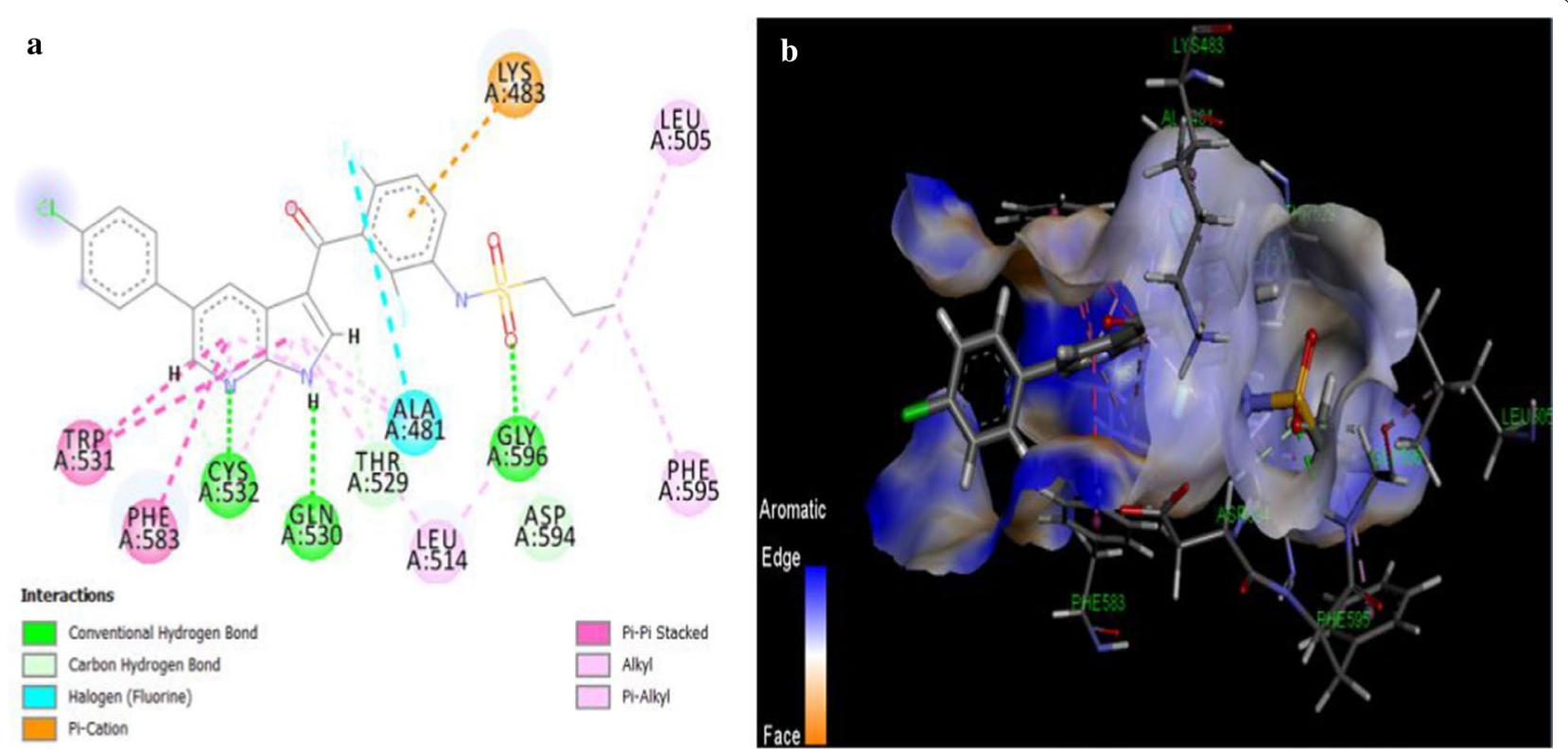

Fig. 6 a 2D diagram for the interaction of vem, with V600E-BRAF, b 3D diagram of vem, in the binding site of V600E-BRAF

this study support our postulation that the compound might inhibit the growth of some melanoma cells via inhibition of V600E-BRAF kinase, in a way similar to vem (Fig. 6).

The complex structure of the docked N3 with the receptor is shown in Fig. 5. It has a MolDock and Rerank score of -166.413 and -141.170 , respectively, while the $\mathrm{E}-\mathrm{H}$ bond was -4.834 (Table 1); this indicates the feasibility of good interactions that exist between this compound and the receptor. There are four $\mathrm{H}$ bond presents between the compound and receptor with ASP594, PHE595, GLY596, and GLY534 residues, respectively. The $\mathrm{N}$-atom of the sulfonamide moiety formed one $\mathrm{H}$ bond with ASP594 residue, and two was observed between O-atom of the sulfonamide moiety to PHE595 and GLY596 residues. The last one was formed between 
Table 3 Predicted drug-likeness based on the Lipinski's rule including bioavailability (BA) and synthetic accessibility (SA) of compound 28, designed compounds and vemurafenib

\begin{tabular}{llllllll}
\hline SN & Mol. wt & HBA & HBD & Log P & TPSA $\left(\AA^{2}\right)$ & BA & SA \\
\hline 28 & 496.310 & 6 & 2 & 4.850 & 98.000 & 0.550 & 3.430 \\
N1 & 545.770 & 6 & 3 & 4.790 & 124.020 & 0.550 & 3.600 \\
N2 & 571.330 & 9 & 2 & 4.650 & 153.050 & 0.170 & 3.930 \\
N3 & 662.460 & 10 & 3 & 5.090 & 198.370 & 0.170 & 4.470 \\
Vem & 489.920 & 6 & 2 & 4.970 & 100.300 & 0.550 & 3.380 \\
\hline
\end{tabular}

Mol. wt. molecular weight, HBA hydrogen bond acceptor, HDB hydrogen bond donor, TPSA topological polar surface area

the $\mathrm{O}$-atom of the $\mathrm{OH}$ group attached to chromene moiety to GLY534 residue as presented in Fig. 4. Five $\mathrm{C}-\mathrm{H}$ bonds with SER535, GLY593, ASP594, GLY596, and HIS539 were also observed. The stability of the complex might be associated with extra alkyl interactions with LEU505, ILE527, LYS483, and ILE527, $\pi$-alkyl interactions with VAL471 (2), ALA481, and LEU514 residues, respectively, and lastly $\pi$-cation interaction with LYS483 similar to vemurafenib (Fig. 6).

The research revealed that $\mathrm{H}$ bond is the main force controlling the interactions that exist between the compound and the protein target and also the interaction energy of the molecules increases with the increase in the number of the hydrogen bonds (Adedirin et al. 2018; Umar et al. 2019). It could be observed that in the $\mathrm{H}$ bond identified with the designed compounds, the number of amino acids involved was found to be better compared to vemurafenib as shown in Figs. 1, 3, 4, 5, and 6, respectively, and there are high similarities. This might acquaint the more reliable binding scores of the chosen molecules for V600E-BRAF. Hence, these novel compounds will serve as good inhibitors of V600E-BRAF showing competitive inhibition with vemurafenib as evident from the molecular docking results.

Moreover, to ensure that the designed compounds are the viable drugs, the drug-likeness and pharmacokinetics ADMET properties were evaluated with vemurafenib as the reference. The SwissADME online tool was used to predict the drug-likeness properties as presented in Table 3, and the pkCSM online tool was adopted in predicting the ADMET properties reported in Table 4. The drug-likeness properties are the main criteria used in screening drugs (candidates) at an initial stage of the drug discovery process (Daina et al. 2017). This method can be described as a means to correlate the physicochemical properties of a given compound with the biopharmaceutical aspect of it in a human body, particularly its influence in oral bioavailability (Bickerton et al. 2012).

Lipinski's rule of five (5) is one of the most popular and helpful guides at the preclinical step of drug development, which predict that if any molecule violates more than two of these criteria $(\mathrm{MW} .<500, \mathrm{HBD} \leq 5, \mathrm{HBA} \leq 10$, $\log p \leq 5$ and TPSA $\left.{ }^{<} 140 \AA^{2}\right)$, the molecule is assumed to be poorly absorbed (Daina et al. 2017). Based on Lipinski's criteria applied, the selected molecules are considered to pass as none violates more than two, as such can be classified as drug-like molecules. Additionally, an evaluation was also conducted using the ABS criteria (Martin 2005), where two of the compounds (compound 28 and N1) have 0.55 as obtained value, while N2 and N3 have a lower value. This criterion was based on a probability value of a molecule to have an optimum profile of permeability and bioavailability, where 0.55 indicates the total obedience of the Lipinski rule of five (Umar et al. 2020) and the rat bioavailability value is $55 \%$, which is a higher probability value than $10 \%$. Moreover, the selected molecules were also assessed for their synthetic accessibility, checking on a scale between 1 (very simple to synthesize) to 10 (very hard and complex to synthesize) (Hadni and Elhallaoui 2020). The synthetic accessibility for all selected molecules is around 3-4 (Table 3), and therefore, they are easy to synthesize.

Further, as for the ADMET properties, a value of $<30 \%$ of an absorbance implies poor absorbance and all the designed compounds showed an absorbance value $>30 \%$, which indicates good absorbance in the human intestine (HI). For the volume of distribution (VDss), a value $>0.45$ is considered to be high. Also, blood-brain barrier (BBB), and the permeability of central nervous system (CNS) standard values are given as $>0.3$ to $<-1 \log \mathrm{BB}$ and $>-2$ to $<-3 \log$ PS, respectively (Pires et al. 2015). Thus, for a given compound, $\log \mathrm{BB}<-1$ indicates a poor distribution of the drug to the brain, while a value of $\log B B>0.3$ implies that the drug can cross the BBB. As for log PS, a value $>-2$ implies that the drug candidate can penetrate the CNS, while a value $<-3$ indicates that it will be difficult for the drug candidate to induce into the CNS (Clark 2003). The results presented in Table 4 indicated that molecules N1 and N3 have shown a high potential to cross barriers compared to vemurafenib.

The metabolism describes the biochemical transformation of a drug candidate by the body. Thus, drugs usually produce several metabolites, which might have different 


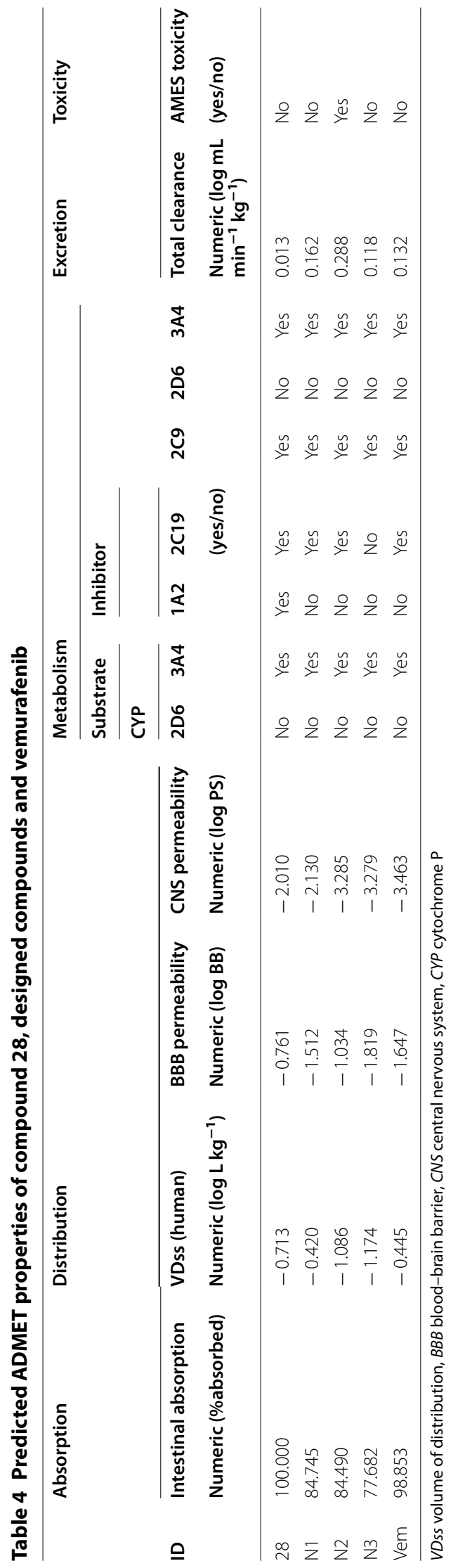


pharmacological and physicochemical properties. It is necessary to consider the metabolism of the drugs, and drug-drug interactions (Kok-Yong and Lawrence 2015). The cytochrome P450 (CYP450) plays a vital role in drug metabolism because it is the main liver protein system involved in oxidation (phase-1 metabolism), as in the case of this research. To date, only 17 CYP families were identified in humans, even though only CYP1, CYP2, CYP3, and CYP4, respectively, involved in the drug metabolism with a CYP (1A2, 2C9, 2C19, 2D6, and 3A4, respectively) were identified to be responsible for the biotransformation of more than $90 \%$ of the drugs that undergo phase-I metabolism (Kok-Yong and Lawrence 2015; Šrejber et al. 2018). Additionally, cytochrome CYP3A4 inhibition is the most vital phenomenon in this research (Thapar 2004). The results presented in Table 4 indicated that the designed compounds are the substrates of CYP3A4 and the inhibitors of CYP3A4, respectively.

Clearance describes the relationship of the drug concentration in the body to the rate of its elimination. Thus, a lower value of the total clearance implies increased persistence of the drugs in the human body, and all the selected molecules showed good persistence in the body for the drug (Yap et al. 2006). Additionally, it is required to investigate whether the designed molecules are nontoxic as this plays a significant role in selecting the best drugs. The results presented in Table 4 indicated that molecules N1 and N3 are non-toxic. The designed compounds N1 and N3 displayed good physicochemical and pharmacokinetic ADMET properties. Thus, according to these results, it can be concluded that these compounds can be used as V600E-BRAF inhibitors and drugs against melanoma cancer in the future.

\section{Conclusion}

The molecular docking studies conducted between V600E-BRAF (PDB ID: 3OG7) and some anticancer compounds were successfully conducted using MVD. All the molecules were found to inhibit the receptor by totally occupying the binding sites in the protein target. The compound with the best docking score for the protein target was utilized to design novel analogues, thereby enhancing the potency of the parent compound. The new compounds developed differ significantly in docking scores from their parent structure as a result of the introduction of some hetero-containing substituents that have the potential to improve the overall docking score by increasing the number of $\mathrm{H}$ bond interactions found in their complex. The docking results of the designed compounds revealed a good MolDock score $(<-90)$, which showed that all the compounds can efficiently bind with the active sites of the target, out of which N1 and N3 were identified as the best hits against V600E-BRAF, as they outperformed vemurafenib, the FDA-approved V600E-BRAF inhibitor. Furthermore, it can be observed that the designed compounds have shown good druglikeness and pharmacokinetics ADMET properties. Therefore, it is recommended that future studies should include the synthesis, in vivo, and ex vivo evaluation of these compounds to validate our hypothesis.

\section{Supplementary information}

Supplementary information accompanies this paper at https://doi. org/10.1186/s42269-020-00432-7.

Additional file 1: Table S1. Chemical Structures of the Studied Flavonebased arylamides. Table S2. Molecular docking results of the studied inhibitors with V600E-BRAF (PDB ID: 3OG7).

\section{Abbreviations}

HTS: High-throughput screening; DFT: Density functional theory; MVD: Molegro Virtual Docker; DS: Discovery Studio; HBA: Hydrogen bond acceptor; HDB: Hydrogen bond donor; TPSA: Topological polar surface area; ADMET: Absorption, distribution, metabolism, excretion, and toxicity.

\section{Acknowledgements}

The authors sincerely acknowledge Ahmadu Bello University, Zaria, for providing the softwares used and all the members of the Physical Chemistry research group for their kind advices and encouragement in the cause of this research. The authors received no direct funding for this research.

\section{Authors' contributions}

AU designed the study. ABU carried out docking simulation and drafted the manuscript. GAS and SU participated in designing the study and edited the manuscript. All authors read and approved the final manuscript.

\section{Funding}

Not applicable.

\section{Availability of data and material}

All data generated or analyzed during this study are included in this published article.

Ethics approval and consent to participate

Not applicable.

Consent for publication

Not applicable.

\section{Competing interests}

The authors declare that they have no competing interests.

Received: 15 September 2020 Accepted: 6 October 2020

Published online: 16 October 2020

\footnotetext{
References

Abdullahi M, Uzairu A, Shallangwa GA, Arthur DE, Umar BA, Ibrahim MT (2020) Virtual molecular docking study of some novel carboxamide series as new anti-tubercular agents. Eur J Chem 11(1):30-36

Adedirin O, Uzairu A, Shallangwa GA, Abechi SE (2018) Optimization of the anticonvulsant activity of 2-acetamido-N-benzyl-2-(5-methylfuran-2-yl) acetamide using QSAR modeling and molecular docking techniques. Beni-Suef Univ J Basic Appl Sci 7(4):430-440

Bickerton GR, Paolini GV, Besnard J, Muresan S, Hopkins AL (2012) Quantifying the chemical beauty of drugs. Nat Chem 4(2):90
} 
Bollag G, Hirth P, Tsai J, Zhang J, Ibrahim PN, Cho H, Spevak W, Zhang C, Zhang Y, Habets G (2010) Clinical efficacy of a RAF inhibitor needs broad target blockade in BRAF-mutant melanoma. Nature 467(7315):596

Borah N, Gunawardana S, Torres H, McDonnell S, Van Slambrouck S (2017) 5, 6, $7,3^{\prime} 4^{\prime}, 5^{\prime}$-Hexamethoxyflavone inhibits growth of triple-negative breast cancer cells via suppression of MAPK and Akt signaling pathways and arresting cell cycle. Int J Oncol 51 (6):1685-1693

Brose MS, Volpe P, Feldman M, Kumar M, Rishi I, Gerrero R, Einhorn E, Herlyn M, Minna J, Nicholson A (2002) BRAF and RAS mutations in human lung cancer and melanoma. Can Res 62(23):6997-7000

Chapman PB, Hauschild A, Robert C, Haanen JB, Ascierto P, Larkin J, Dummer R, Garbe C, Testori A, Maio M (2011) Improved survival with vemurafenib in melanoma with BRAF V600E mutation. N Engl J Med 364(26):2507-2516

Cheng T, Li Q, Zhou Z, Wang Y, Bryant SH (2012) Structure-based virtual screening for drug discovery: a problem-centric review. AAPS J 14(1):133-141

Choi W-K, El-Gamal MI, Choi HS, Baek D, Oh C-H (2011) New diarylureas and diarylamides containing 1, 3, 4-triarylpyrazole scaffold: synthesis, antiproliferative evaluation against melanoma cell lines, ERK kinase inhibition, and molecular docking studies. Eur J Med Chem 46(12):5754-5762

Clark DE (2003) In silico prediction of blood-brain barrier permeation. Drug Discov Today 8(20):927-933

Daina A, Michielin O, Zoete V (2017) SwissADME: a free web tool to evaluate pharmacokinetics, drug-likeness and medicinal chemistry friendliness of small molecules. Sci Rep 7:42717

Dhillon AS, Hagan S, Rath O, Kolch W (2007) MAP kinase signalling pathways in cancer. Oncogene 26(22):3279

Elnaggar MH, Abushouk Al, Hassan AH, Lamloum HM, Benmelouka A, Moatamed SA, Abd-Elmegeed H, Attia S, Samir A, Amr N (2019) Nanomedicine as a putative approach for active targeting of hepatocellular carcinoma. In: Seminars in cancer biology. Elsevier

Gray-Schopfer V, Wellbrock C, Marais R (2007) Melanoma biology and new targeted therapy. Nature 445(7130):851

Hadni H, Elhallaoui M (2020) 2D and 3D-QSAR, molecular docking and ADMET properties in silico studies of azaaurones as antimalarial agents. New J Chem 44(16):6553-6565

Hassan AH, Lee K-T, Lee YS (2020) Flavone-based arylamides as potential anticancers: design, synthesis and in vitro cell-based/cell-free evaluations. Eur J Med Chem 187:111965

Kok-Yong S, Lawrence L (2015) Drug distribution and drug elimination. In: Ahmed TA (ed) Basic pharmacokinetic concepts and some clinical applications. Intech, Rijeka, pp 99-116

Kudchadkar R, Paraiso KH, Smalley KS (2012) Targeting mutant BRAF in melanoma: current status and future development of combination therapy strategies. Cancer J (Sudbury, Mass.) 18(2):124

Landucci E, Gencarelli M, Mazzantini C, Laurino A, Pellegrini-Giampietro DE, Raimondi L (2019) N-(3-Ethoxy-phenyl)-4-pyrrolidin-1-yl-3-trifluoromethyl-benzamide (EPPTB) prevents 3-iodothyronamine (T1AM)-induced neuroprotection against kainic acid toxicity. Neurochem Int 129:104460

Luo C, Xie P, Marmorstein R (2008) Identification of BRAF inhibitors through in silico screening. J Med Chem 51(19):6121-6127

Martin YC (2005) A bioavailability score. J Med Chem 48(9):3164-3170

Martinez-Mayorga K, Madariaga-Mazon A, Medina-Franco JL, Maggiora G (2020) The impact of chemoinformatics on drug discovery in the pharmaceutical industry. Expert Opin Drug Discov 15(3):293-306

Molegro A (2011) MVD 5.0 Molegro virtual docker. DK-8000 Aarhus C, Denmark
Pires DE, Blundell TL, Ascher DB (2015) pkCSM: predicting small-molecule pharmacokinetic and toxicity properties using graph-based signatures. J Med Chem 58(9):4066-4072

Robinson SD, O'Shaughnessy JA, Cowey CL, Konduri K (2014) BRAF V600Emutated lung adenocarcinoma with metastases to the brain responding to treatment with vemurafenib. Lung Cancer 85(2):326-330

Roskoski R (2012) MEK1/2 dual-specificity protein kinases: structure and regulation. Biochem Biophys Res Commun 417(1):5-10

Salton M, Kasprzak WK, Voss T, Shapiro BA, Poulikakos PI, Misteli T (2015) Inhibition of vemurafenib-resistant melanoma by interference with pre-mRNA splicing. Nat Commun 6(1):1-7

Schmidt T, Bergner A, Schwede T (2014) Modelling three-dimensional protein structures for applications in drug design. Drug Discov Today 19(7):890-897

Šrejber M, Navrátilová V, Paloncýová M, Bazgier V, Berka K, Anzenbacher P, Otyepka M (2018) Membrane-attached mammalian cytochromes P450: an overview of the membrane's effects on structure, drug binding, and interactions with redox partners. J Inorg Biochem 183:117-136

Thapar MM (2004) Pharmacokinetics and dynamics of atovaquone and proguanil (Malarone R). Institutionen för medicin/Department of Medicine

Thomsen R, Christensen MH (2006) MolDock: a new technique for high-accuracy molecular docking. J Med Chem 49(11):3315-3321

Umar BA, Uzairu A, Shallangwa GA, Uba S (2019) Rational drug design of potent V600E-BRAF kinase inhibitors through molecular docking simulation. J Eng Exact Sci 5(5):0469-0481

Umar AB, Uzairu A, Shallangwa GA, Uba S (2020) In silico evaluation of some 4-(quinolin-2-yl) pyrimidin-2-amine derivatives as potent V600E-BRAF inhibitors with pharmacokinetics ADMET and drug-likeness predictions. Future J Pharm Sci 6(1):1-10

Verma AK, Pratap R (2010) The biological potential of flavones. Nat Prod Rep 27(11):1571-1593

Vu HN, Kim JY, Hassan AH, Choi K, Park J-H, Park KD, Lee JK, Pae AN, Choo H, Min S-J (2016) Synthesis and biological evaluation of picolinamides and thiazole-2-carboxamides as mGluR5 (metabotropic glutamate receptor 5) antagonists. Bioorg Med Chem Lett 26(1):140-144

Wan PT, Garnett MJ, Roe SM, Lee S, Niculescu-Duvaz D, Good VM, Project CG, Jones CM, Marshall CJ, Springer CJ (2004) Mechanism of activation of the RAF-ERK signaling pathway by oncogenic mutations of B-RAF. Cell 116(6):855-867

Yap C, Li Z, Chen Y (2006) Quantitative structure-pharmacokinetic relationships for drug clearance by using statistical learning methods. J Mol Graph Model 24(5):383-395

Zhang X, Li H, Zhang H, Liu Y, Huo L, Jia Z, Xue Y, Sun X, Zhang W (2017) Inhibition of transmembrane member $16 \mathrm{~A}$ calcium-activated chloride channels by natural flavonoids contributes to flavonoid anticancer effects. $\mathrm{Br} J$ Pharmacol 174(14):2334-2345

Zhao L, Yuan X, Wang J, Feng Y, Ji F, Li Z, Bian J (2019) A review on flavones targeting serine/threonine protein kinases for potential anticancer drugs. Bioorg Med Chem 27:677-685

\section{Publisher's Note}

Springer Nature remains neutral with regard to jurisdictional claims in published maps and institutional affiliations. 